\title{
Ultrasound guided balloon catheterisation: a new method of fetal lower urinary tract obstruction management
}

\author{
Marzena Dębska' ${ }^{1}$, Adam Koleśnik² ${ }^{2}$ Piotr Kretowicz' \\ Anna Olędzka ${ }^{1}$, Piotr Gastoł ${ }^{3}$, Romuald Dębski ${ }^{1}$ \\ ${ }^{1}$ II Department of Obstetrics and Gynecology, Medical Center of Postgraduate Education (CMKP), Warsaw, Poland \\ ${ }^{2}$ Cardiac Catheterisation Laboratory, Children's Memorial Health Institute, Warsaw, Poland \\ ${ }^{3}$ Department of Pediatric Urology, Children's Memorial Health Institute, Warsaw, Poland
}

\begin{abstract}
Objectives: Fetal lower urinary tract obstruction (LUTO), most often associated with presence of posterior urethral valves, poses high risk of perinatal mortality or postnatal renal failure. Looking for a method of causative treatment we have developed a technique of fetal urethroplasty with a coronary angioplasty balloon catheter inserted under an ultrasonographic guidance via an 18-gauge needle introduced transabdominally to fetal bladder.

Material and methods: We have used this procedure in three women with singleton pregnancies (two primiparas and one multipara, 32-35 years of age), diagnosed with fetal megacystis at 12-16 weeks of gestation. Urethral catheterization was carried out at 16-18 weeks and an unobstructed urine flow was achieved in all three cases immediately after the procedure, followed by a resolution of megacystis and normalization of amniotic fluid volume.

Results: In all three cases, the post-procedure period was uneventful. In the first two fetuses, amniotic fluid volume remained normal until 30 weeks of gestation when a gradual development of oligohydramnios and some signs of renal cystic dysplasia were observed. Nevertheless, both pregnancies were continued till term (37 and 39 weeks, respectively) and two boys without signs of pulmonary hypoplasia were delivered. The third patient is currently 25 weeks pregnant; volume of amniotic fluid in her fetus is normal and no signs of urinary flow obstruction or renal dysplasia have been recorded thus far.

Conclusions: Although some technical aspects of the procedure still need to be established, it seems worth consideration as a form of potentially least traumatic intrauterine intervention in fetuses with lower urinary tract obstruction.
\end{abstract}

Key words: posterior urethral valves, balloon urethroplasty, intrauterine treatment, megacystis, renal dysplasia

Ginekologia Polska 2017; 88, 5: 255-259

\section{INTRODUCTION}

Fetal lower urinary tract obstruction (LUTO) is a group of congenital conditions diagnosed with a frequency of approximately 2.2 per 10000 births. Two most common causes of LUTO are posterior urethral valves (PUVs) and urethral atresia. Other isolated malformations include anterior urethral valves and urethral stenosis/hypoplasia. LUTO may be also a component of various congenital syndromes, such as megacystis-microcolon syndrome, megacystis-megaureter syndrome, prune-belly syndrome and chromosome 18 ,
13 and 21 aneuploidy. LUTO causes an increase in urinary pressure, which may impede the development of collecting ducts, tubules and glomeruli, and eventually results in renal dysplasia. Lack of urine output leads to oligohydramnios, pulmonary hypoplasia, Potter sequence and preterm birth [1-4]. LUTO is associated with high mortality or high risk of renal failure in the childhood [5]. Neonates with complete PUVs and anhydramnios usually die shortly after birth due to pulmonary hypoplasia. Urine output and amniotic fluid volume in fetuses with uncomplete PUVs may be initially 
normal or only slightly reduced; however, urinary pressure is still elevated, which may contribute to gradual kidney damage and renal insufficiency after birth.

LUTO is usually diagnosed during routine ultrasound in the second trimester, except from the most severe forms which are detected already in the first trimester [6]. Typical symptoms of LUTO include megacystis with a keyhole sign, bilateral hydronephrosis with or without cystic renal changes, and oligohydramnios or anhydramnios. Available management options include expectant management, termination of pregnancy or intrauterine intervention. Unfortunately, we lack an accurate method to identify fetuses that may really benefit from in utero treatment, and in many cases renal damage at the time of intervention is too advanced to prevent further deterioration of kidney function [7]. A few prenatal procedures can be offered to fetuses with LUTO [8], with vesicoamniotic shunting (VAS) being the standard treatment. Also successful attempts to perform open fetal vesicostomy or fetal cystoscopic laser ablation of the valves have been reported. Ruano et al. [9] and Hofmann et al. [10] presented their single experience with fetoscopic retrograde catheterization of fetal urethra with a guidewire and transurethral stent placement. In this report, we present three cases of LUTO treated by dilatation of fetal urethra with a coronary balloon catheter under an ultrasonographic guidance. To the best of our knowledge, this kind of intrauterine intervention has not been reported thus far.

\section{MATERIAL AND METHODS}

Patient \#1, a 32-year-old primipara was referred to hospital at 17 weeks of gestation with the diagnosis of megacystis and oligohydramnios. Despite a slight increase in renal echogenicity, no distention was observed in either the renal pelvis or calyces. The keyhole sign, typical ultrasonographic manifestation of PUVs was documented, with distended fetal bladder reaching up to $64 \mathrm{~mm}$ in height. Patient \#2, a 34-year-old primipara was admitted at 16 weeks of gestation. Also in this case, an evident fetal megacystis (bladder height $41 \mathrm{~mm}$ ) was detected, along with the keyhole sign and slight bilateral ureteral dilation. Similar to the first case, no ultrasonographic abnormalities of the kidneys were found except from a slight increase in echogenicity. Amniotic fluid volumes in both patients were at lower normal limit. In Patient \#1, ultrasonographic evidence of fetal megacystis was found at 11-14 weeks scan. Patient \#2 did not undergo an ultrasonographic exam prior to 16 weeks of gestation. Patient \#3 was a 35-year-old woman in her fourth pregnancy, with a history of two miscarriages and one termination due to trisomy 21. Ultrasonographic findings at 11-14 weeks scan were normal, and the first evidence of megacystis emerged at 16.4 weeks of gestation. At the time of diagnosis, urinary bladder had $34 \mathrm{~mm}$ in length. The kidneys were slightly hyperechoic with a mild hydronephrosis (normal AP diameter of renal pelvis but with an evident distention of the calyces). Amniotic fluid volume was at lower normal limit. Aside from club foot deformity in fetus from Patient \#1, no other ultrasonographic and echocardiographic anomalies were found, and amniocentesis and genetic analysis $(\mathrm{aCGH})$ demonstrated presence of normal $46, X Y$ fetal karyotype in all cases.

Upon being informed about prognosis and available management options, all pregnant women consented for balloon catheter dilatation procedure.

The procedure was carried out under maternal local anesthesia and fetal anesthesia with fentanyl and atracurium (30 $\mu \mathrm{g} / \mathrm{kg}$ estimated fetal weight [EFW] and $0.2 \mathrm{mg} / \mathrm{kg}$ EFW, respectively, injected to fetal gluteal muscle). Fetal urethra was dilated with a Maverick 2 balloon catheter (the diameter of the leader of the catheter is 0,014 " and diameter of the balloon is $2 \mathrm{~mm}$, length $9 \mathrm{~mm}$, the crossing profile of un uninflated balloon is less then $1 \mathrm{~mm}$ ) inserted under an ultrasonographic guidance. In Patient \#1, the intervention took place at $17+3$ weeks of gestation. An 18-gauge (G) needle, introduced to fetal bladder transabdominally, was used to insert the coronary catheter to the urethra. After positioning the catheter, the balloon was inserted. Although the fetus was in a cephalic position, the exact location of the catheter in the urethra could not be visualized clearly due to maternal obesity and anterior location of the placenta. Owing these technical difficulties and small size of fetal urethra, the balloon was inserted and retracted a few times instead of being inflated (diameter of the balloon is larger than the catheter's diameter). In Patient \#2, the procedure was carried out at $16+5$ weeks of gestation, using the same protocol of anesthesia as described above. Due to an unfavorable position of the fetus, with pelvis close to the internal os of the cervix, a transvaginal transducer was used after transabdominal insertion of the needle to fetal bladder. Then, similarly the guide wire was inserted to fetal urethra, followed by a coronary balloon catheter. In Patient \#3, the procedure was carried out at 18.1 weeks of gestation. During nearly two weeks between the diagnosis of LUTO and the intervention, the status of fetal urinary tract has deteriorated considerably. Prior to the procedure, the bladder was $61 \mathrm{~mm}$ in length, AP diameter of renal pelvis equaled to $5 \mathrm{~mm}$, and evidently distended renal calyces could be observed along with bilateral megaureters. Similar to previously described cases, an 18-G needle was inserted via uterine wall into the fetal bladder under a transabdominal ultrasonographic guidance (Fig. 1), and then the procedure was monitored using a transvaginal ultrasound. A balloon catheter was inserted to fetal urethra, reaching down to the amniotic cavity (Figs. 2, 3). The balloon was slightly inflated manually under an ultrasonographic guidance in the three 


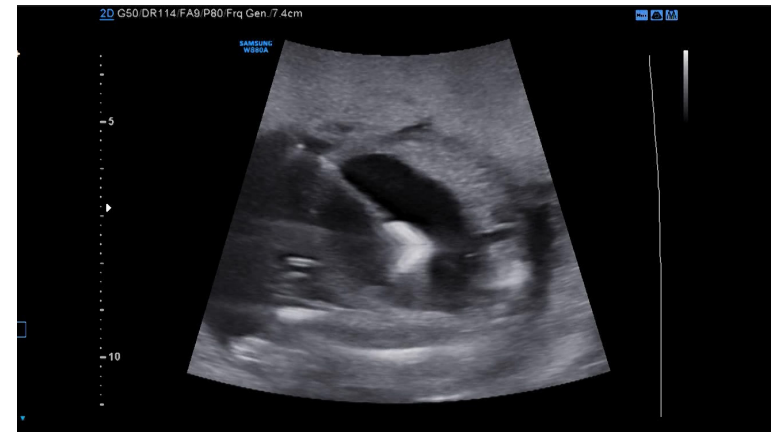

Figure 1. 18-G needle in fetal megacystis (Patient \#3)

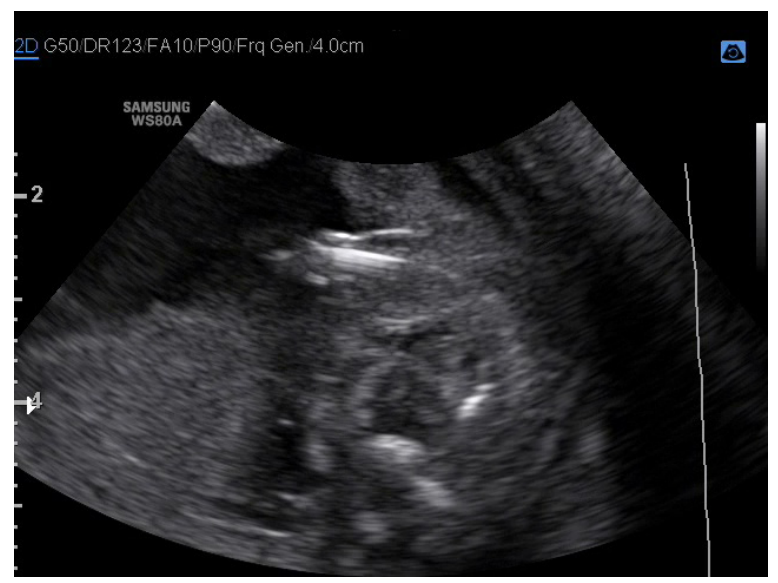

Figure 3. Balloon catheter in fetal penile urethra (Patient \#3)

different segments of the urethra (Fig. 4), corresponding to entire length of the urethra, and then removed.

\section{RESULTS}

An unobstructed urine flow was observed in all three cases immediately after the procedure, followed by a resolution of megacystis. Control ultrasonographic exam performed one day later confirmed decompression of thick-walled urinary bladder and normal volume of amniotic fluid. The post-procedure period was uneventful in all three cases.

In fetus from Patient \#1, amniotic fluid volume remained normal until 30 weeks of gestation, when the first ultrasonographic evidence of oligohydramnios emerged on a control ultrasound, along with the signs of progressive renal cystic dysplasia. Male newborn was delivered vaginally at 39 weeks of gestation with a 7-point Apgar score, which reflected presence of transient respiratory problems. During the first day of life, until normalization of respiratory function, the neonate required mechanical ventilation in bi-pap. Ultrasonographic exam revealed bilateral renal dysplasia, without parenchymatous-medullar differentiation, as well as the presence of tiny cysts in the left kidney. Urine output was normal (1.6-2.6 mL/kg/hour), and no signs of infection were

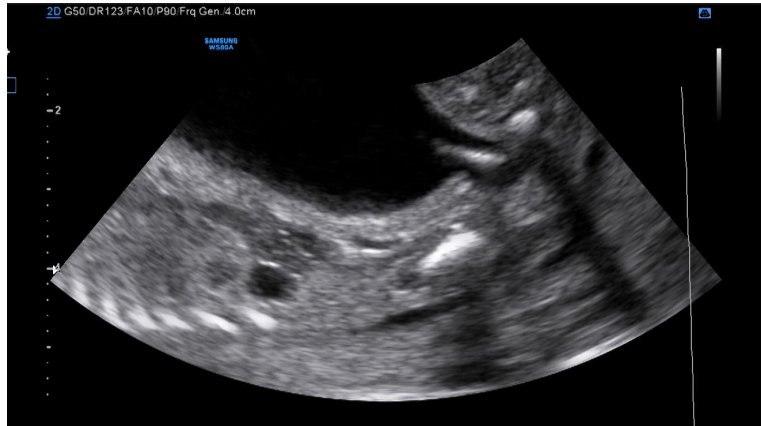

Figure 2. Balloon catheter in proximal fetal urethra (Patient \#3)

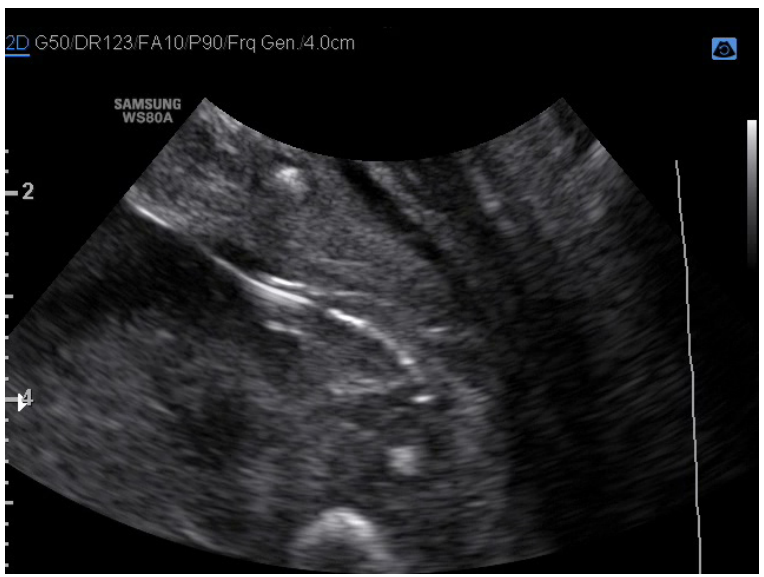

Figure 4. Inflated balloon in fetal urethra (Patient \#3)

observed. Serum creatinine was $0.7 \mathrm{mg} / \mathrm{dL}$ on the first day of life, but then raised to $3.7 \mathrm{mg} / \mathrm{dL}$. An urethrocystoscopy performed at the Urology Department confirmed presence of residual PUVs without urine flow obstruction. However, forced diuresis, antibiotic therapy, natrium bicarbonate and vitamin D supplementation were introduced due to the evidence of renal dysplasia. Further, the club deformity in the right foot has been corrected. The neonate was discharged from the Pediatric Urology Department at one month of age, in a good general condition, with serum creatinine $2.3 \mathrm{mg} / \mathrm{dL}$. Vitamin D and calcium supplementation were prescribed and further follow-up at a nephrology and urology unit was recommended. Serum creatinine increased up to $3.4 \mathrm{mg} / \mathrm{dL}$ at 8 months of age, which constituted an indication to dialysis therapy. Currently, at 15 months of age, the boy urinates independently but is also is still subjected to peritoneal dialyses (2-3 times weekly) and his serum creatinine approximates $5.4 \mathrm{mg} / \mathrm{dL}$. Meanwhile, he underwent a cryptorchidism surgery, and awaits a kidney transplant. Otherwise he develops normally. Owing retained function of the bladder, he is scheduled for kidney transplantation to his own bladder, which is the most comfortable option to provide him with normal quality of life in the future. 
Further course of pregnancy in Patient \#2 was uneventful as well. Amniotic fluid volume remained normal until 30 weeks of gestation when a gradual development of oligohydramnios and signs of renal cystic dysplasia were observed. Male neonate was delivered via the cesarean at 37 weeks of gestation, with 8-9-10-point Apgar score. The decision to perform the cesarean was driven by the evidence of fetal asphyxia during the course of vaginal birth. Ultrasonography confirmed presence of small, hypoechogenic kidneys with a parenchymatous cyst and bilateral ureter distention. Laboratory tests revealed elevated levels of inflammatory markers and pyuria; serum creatinine amounted to $2.8 \mathrm{mg} / \mathrm{dL}$. Third-generation cephalosporine was implemented due to presence of Escherichia coli and Klebsiella pneumoniae infection. The neonate underwent a cystoscopy with transurethral incision (TUI) of residual PUVs and cervix of the bladder at Urology Department. The boy was discharged home at 3 weeks of age, in a good general condition, with serum creatinine level of $1.4 \mathrm{mg} / \mathrm{dL}$, and recommendation of further follow-up at a nephrology and urology unit. Unfortunately, he required creation of an uretero-cutaneous fistula due to presence of persistent megaureter on the left side. Currently, at 7 months of age, the boy urinates freely and presents with normal serum creatinine $(0.9 \mathrm{mg} / \mathrm{dL})$ and does not require a renal replacement therapy. Noticeably, fetuses from Patients \#1 and \#2 were delivered at term, and the cesarean section in Patient \#2 was necessary solely due to obstetrical indications. Patient \#3 is currently 27 weeks pregnant; volume of amniotic fluid in her fetus is normal and no signs of urinary flow obstruction has been recorded thus far.

\section{DISCUSSION}

Treatment of fetal LUTO remains an unresolved and widely discussed problem of prenatal therapy. Prenatal interventions in fetuses with LUTO, aimed at prevention or at least reduction of postnatal morbidity and mortality, date back to 1982, when Harrison et al. [11] reported a case of severe fetal bilateral hydronephrosis treated by ureterostomy. The outcomes of the intrauterine treatment of LUTO has improved since then, and some techniques, such as vesicoamniotic shunting (VAS), fetoscopic laser ablation of the PUVs and even a cystoscopic transurethral stent placement have been implemented. Long-term postnatal outcomes in children after intrauterine VAS were a subject of many previous studies. Freedman et al. [12] and Holmes et al. [13] performed this procedure in 34 and 14 fetuses with LUTO, respectively, with $60 \%$ and $57 \%$ survival rates, and normal kidney function in $43 \%$ and $37 \%$ of the cases, respectively. These findings are consistent with the results of other studies in which VAS resulted in normalization of fluid volume and normal renal function in only $50 \%$ [14] and $45 \%$ of neonates [15]. PLUTO trial was originally designed to include 150 women with singleton pregnancies with ultrasonographic evidence of LUTO, randomized to either VAS or conservative management. However, only 31 patients have been eventually recruited; after a 1-year follow-up, only 2/7 neonates subjected to VAS showed no evidence of renal dysfunction [16].To summarize, most findings imply that VAS neither eliminates the cause of LUTO nor effectively prevents the resultant renal damage. It has many disadvantages, like incorrect placement, displacement, urinary ascites formation but probably the most important is that it affects bladder function because the presence of bladder shunt precludes a normal urination cycle. The bladder is spastic after birth and causes an increase in urinary pressure which often contributes to vesicoureteral reflux and secondary hydronephrosis.

An attempt to resolve the issue of fetal PUVs is their fetoscopic laser fulguration. This procedure was first performed by Sananes et al. [17] in 23 fetuses with LUTO; $60.9 \%$ of them survived (14/23) and $85.7 \%(12 / 14)$ of them had normal renal function after birth. The only documented procedure of cystoscopic transurethral stent placement was performed by Ruano et al. [9] at 22 weeks of gestation; currently, their patient is at 5 years of age and still presents with normal renal function. The main technical drawback of fetal cystoscopy are difficulties in reaching posterior urethra, since generally sharp vesicourethral angle becomes even sharper with gestational age. Therefore, even if posterior urethra can be reached, urethral valves may not be adequately visualized. This represents a serious limitation of fetal cystoscopy and potential cause of significant morbidity, such as fistula formation or inadvertent thermal damage to surrounding tissues. Up to $10 \%$ of newborns subjected to cystoscopy in utero presented with urological fistulas at birth [17]. Furthermore, long-term complications of intrauterine cystoscopy may potentially interfere with sexual life and fertility in the adulthood. All this problems with prenatal LUTO management justifies further research on causative and less invasive intrauterine treatment of LUTO.

To overcome the technical limitations of fetoscopic procedure and to avoid the complications inherent either to fetoscopy or to VAS, we have developed our own technique of fetal urethroplasty with a coronary angioplasty balloon catheter. The first advantage of this technique is its less invasive character. Further, the whole procedure is carried out under an ultrasonographic guidance, which may provide an excellent visualization of the coronary catheter as well as the fetal bladder and the urethra. Secondly, the flexible and trackable catheter can be easily diverted into all directions and inserted into even very narrow and tight spaces. This is exactly the same type of catheter which we use during fetal balloon cardiac valvuloplasties at our center [18-20]. It can be introduced into the valve with down to $1 \mathrm{~mm}$ in 
diameter, because the diameter of the leader of the catheter is only $0.014^{\prime \prime}(0.35 \mathrm{~mm})$.

To this date, a total of three procedures of balloon catheterization have been carried out at our center in fetuses with suspected isolated PUVs. The treatment produced satisfactory outcomes regarding intrauterine relieving of the obstruction; as a result of restoring urinary patency, none of the fetuses presented with megacystis or hydronephrosis till the end of the pregnancy. Unfortunately, despite successful catheterization of the urethra, progressive oligohydramnios and cystic renal dysplasia have been observed in two fetuses in late pregnancy, perhaps as a consequence of earlier irreversible damage to kidney tissue. Patient \#3 is still pregnant but her fetus shows no signs of lower urinary tract obstruction and the kidneys look normally. To the best of our knowledge, this is the first report documenting ultrasound-guided insertion of a balloon catheter to fetal urethra as an intrauterine approach to fetal megacystis. Although the efficacy and technical aspects of the procedure still need to be established, it seems to be an option of fetal PUV treatment worth consideration. Intrauterine catheterization restores patency of lower urinary tract without a damage to fetal urethra and surrounding tissues. Furthermore, in contrast to VAS, this procedure does not interfere with normal micturition and urinary bladder function during the pregnancy. Since fetal urethra is dilated only mechanically and the catheter is removed immediately after the procedure, there is no risk of thermal destruction or long term irritation of surrounding tissues.

\section{REFERENCES}

1. Lissauer D, Morris RK, Kilby MD. Fetal lower urinary tract obstruction. Semin Fetal Neonatal Med. 2007; 12(6): 464-470, doi: 10.1016/j. siny.2007.06.005, indexed in Pubmed: 17761463.

2. Wu S, Johnson MP. Fetal lower urinary tract obstruction. Clin Perinatol. 2009; 36(2): 377-390, doi: 10.1016/j.clp.2009.03.010, indexed in Pubmed: 19559326.

3. Tonni G, Vito I, Ventura A, et al. Fetal lower urinary tract obstruction and its management. Arch Gynecol Obstet. 2013; 287(2): 187-194, doi: 10.1007/s00404-012-2615-9, indexed in Pubmed: 23138754

4. Clayton DB, Brock JW. Lower urinary tract obstruction in the fetus and neonate. Clin Perinatol. 2014; 41(3): 643-659, doi: 10.1016/j. clp.2014.05.012, indexed in Pubmed: 25155733.
5. Ruano R, Safdar A, Au J, et al. Defining and predicting, intrauterine fetal renal failure' in congenital lower urinary tract obstruction. Pediatr $\mathrm{Ne}-$ phrol. 2016; 31(4): 605-612, doi: 10.1007/s00467-015-3246-8, indexed in Pubmed: 26525197.

6. Ruano R, Yoshisaki CT, Salustiano EMA, et al. Early fetal cystoscopy for first-trimester severe megacystis. Ultrasound Obstet Gynecol. 2011; 37(6): 696-701, doi: 10.1002/uog.8963, indexed in Pubmed: 21337440.

7. Morris RK, Quinlan-Jones E, Kilby MD, et al. Systematic review of accuracy of fetal urine analysis to predict poor postnatal renal function in cases of congenital urinary tract obstruction. Prenat Diagn. 2007; 27(10): 900-911, doi: 10.1002/pd.1810, indexed in Pubmed: 17610312.

8. Agarwal SK, Fisk NM. In utero therapy for lower urinary tract obstruction. Prenat Diagn. 2001; 21(11): 970-976, indexed in Pubmed: 11746151.

9. Ruano R, Yoshizaki CT, Giron AM, et al. Cystoscopic placement of transurethral stent in a fetus with urethral stenosis. Ultrasound Obstet Gynecol. 2014; 44(2): 238-240, doi: 10.1002/uog.13293, indexed in Pubmed: 24375864.

10. Hofmann R, Becker T, Meyer-Wittkopf M, et al. Fetoscopic placement of a transurethral stent for intrauterine obstructive uropathy. J Urol. 2004; 171(1): 384-386, doi: 10.1097/01.ju.0000102933.25905.08, indexed in Pubmed: 14665937

11. Harrison MR, Golbus MS, Filly RA, et al. Fetal surgery for congenital hydronephrosis. N Engl J Med. 1982; 306(10): 591-593, doi: 10.1056/ /NEJM198203113061006, indexed in Pubmed: 7057815.

12. Freedman $A L$, Johnson MP, Smith $C A$, et al. Long-term outcome in children after antenatal intervention for obstructive uropathies. Lancet. 1999; 354(9176): 374-377, doi: 10.1016/S0140-6736(98)11006-1, indexed in Pubmed: 10437866.

13. Holmes N, Harrison MR, Baskin LS. Fetal surgery for posterior urethral valves: long-term postnatal outcomes. Pediatrics. 2001; 108(1): E7, indexed in Pubmed: 11433086.

14. McLorie G, Farhat W, Khoury A, et al. Outcome analysis of vesicoamniotic shunting in a comprehensive population. J Urol. 2001; 166(3): 1036-1040, indexed in Pubmed: 11490292.

15. Biard JM, Johnson MP, Carr MC, et al. Long-term outcomes in children treated by prenatal vesicoamniotic shunting for lower urinary tract obstruction. Obstet Gynecol. 2005; 106(3): 503-508, doi: 10.1097/01. AOG.0000171117.38929.eb, indexed in Pubmed: 16135579.

16. Morris RK, Malin GL, Quinlan-Jones E, et al. Percutaneous vesicoamniotic shunting in Lower Urinary Tract Obstruction (PLUTO) Collaborative Group. Percutaneous vesicoamniotic shunting versus conservative management for fetal lower urinary tract obstruction (PLUTO): a randomised trial. Lancet. 2013; 382(9903): 1496-1506, doi: 10.1016/S01406736(13)60992-7, indexed in Pubmed: 23953766.

17. Sananes N, Favre R, Koh CJ, et al. Urological fistulas after fetal cystoscopic laser ablation of posterior urethral valves: surgical technical aspects. UItrasound Obstet Gynecol. 2015; 45(2): 183-189, doi: 10.1002/uog.13405, indexed in Pubmed: 24817027.

18. Dangel J, Debska M, Koleśnik A, et al. [The first successful fetal aortic balloon valvuloplasty in Poland]. Ginekol Pol. 2011; 82(8): 632-636, indexed in Pubmed: 21957611.

19. Dangel J, Dębska M, Koleśnik A, et al. Successful aortic valvuloplasties in fetuses - the new era and new challenge for Polish perinatal cardiology. Echo Płodu. 2012; 2: 16-20.

20. Dębska M, Szymkiewicz-Dangel J, Koleśnik A, et al. [Fetal cardiac interventions - are we ready for them?]. Ginekol Pol. 2015; 86(4): 280-286, indexed in Pubmed: 26117987. 